\title{
Occupations anciennes des plateaux miniers calédoniens à Thio et à Tontouta : premières prospections et datations
}

Christophe Sand, Jacques Bolé, André-John OUETCHO, Yves-Béalo GONY et David BARET

\section{(2) OpenEdition \\ Journals}

\section{Édition électronique}

URL : http://journals.openedition.org/jso/6582

DOI : $10.4000 /$ jso.6582

ISSN : $1760-7256$

Éditeur

Société des océanistes

Édition imprimée

Date de publication : 30 juin 2012

Pagination : $31-44$

ISBN : 978-2-85430-032-1

ISSN : 0300-953x

Référence électronique

Christophe Sand, Jacques Bolé, André-John OUETCHO, Yves-Béalo GONY et David BARET, «

Occupations anciennes des plateaux miniers calédoniens à Thio et à Tontouta : premières

prospections et datations », Journal de la Société des Océanistes [En ligne], 134 | 1er semestre 2012,

mis en ligne le 12 juillet 2012, consulté le 19 avril 2019. URL : http://journals.openedition.org/jso/6582 ; DOI : $10.4000 /$ jso.6582 


\title{
Occupations anciennes des plateaux miniers calédoniens à Thio et à Tontouta : premières prospections et datations
}

\author{
par \\ Christophe SAND, Jacques BOLÉ, André-John OUETCHO, Yves-Béalo GONY \\ et David BARET*
}

\section{RÉSUMÉ}

L'étude archéologique des massifs miniers de la Grande Terre calédonienne ne s'est réellement développée qu'au cours de la dernière décennie, suite aux études d'impact imposées aux aménageurs. Des inventaires partiels menés sur deux zones de mines, le Camp des Sapins (Thio) et le fond de la vallée de la Tontouta, sont présentés. La première réalisation d'une série de datations au ${ }^{14} \mathrm{C}$ a permis de démontrer, en complément des identifications de poteries anciennes, qu'une partie des aménagements découverts se rapporte à la période préeuropéenne, avec une occupation principalement au cours du deuxième millénaire après $J$-C., lors de voyages entre les deux côtes de la Grande Terre, mais probablement également pour la réalisation de rituels. Ces résultats viennent éclairer d'un jour nouveau l'histoire ancienne des ensembles miniers calédoniens, souvent considérés comme des "espaces vacants", d'où la nécessité de mieux connaître les différentes formes d'utilisation de ces massifs stériles par les groupes kanak précoloniaux, en lien avec les revendications autochtones actuelles adressées aux opérateurs miniers.

Mots-CLÉs : Nouvelle-Calédonie, massifs miniers, archéologie, datations, installations kanak, revendications

La Grande Terre de Nouvelle-Calédonie a développé au cours de son histoire géologique un faciès et des caractéristiques environnementales

\begin{abstract}
Archaeological studies on the mining regions on New Caledonia's main island have only really started in recent times, as part of impact assessments imposed to mining companies. This paper presents the outcome of partial surveys fulfilled in two mining regions, respectively the Camp des Sapins of Thio and the upper valley of Tontouta. Through the first-ever ${ }^{14} \mathrm{C}$ dating of a number of samples, it is possible to demonstrate, aside from the identification of old pottery types, that part of the structures discovered are related to pre-European times, with an occupation mainly in the second millennium $A D$, during voyages between the two coasts of the Island but also probably for ritual performances. These results shed new light on the old history of New Caledonia's mining regions, often seen as mere "empty environments". They show the need to better understand the use made of these sterile lands by the pre-colonial Kanak groups, in relation to present-day claims addressed by indigenous groups to mining companies across the world.
\end{abstract}

KeYwords: New Caledonia, mining regions, archaeology, dating, Kanak occupations, claims

uniques dans le Pacifique. Issue de la séparation d'un fragment de Gondwana il y a quelque 80 millions d'années, la dorsale formant aujour-

\footnotetext{
* Institut d'archéologie de la Nouvelle-Calédonie et du Pacifique (IANCP), christophe.sand@iancp.nc, jacques.bolé@iancp.nc, andre-john.ouetcho@iancp.nc, yves-bealo.gony@iancp.nc, david.baret@iancp.nc
} 
d'hui l'axe occidental de la Nouvelle-Calédonie a émergé il y a environ 45 millions d'années, après une période d'immersion océanique. $\mathrm{Ce}$ processus de surrection s'est accompagné du glissement d'une partie de la plaque océanique sur la dorsale il y a plus de 30 millions d'années, avant un processus d'érosion qui a abouti à la mise en place d'une croûte métamorphique sur à peu près un tiers de la superficie totale de la Grande Terre (Paris, 1981). Ce sont ces sols ultrabasiques (péridotites) riches en nickel, en fer et en chrome qui ont au cours des derniers 140 ans fait la principale richesse économique de l'archipel calédonien, dans le cadre d'exploitations minières intensives développées sur tous les grands ensembles exploitables.

Durant la période coloniale et jusqu'à ces dernières décennies, les exploitations minières, en Nouvelle-Calédonie comme ailleurs dans le monde, se sont faites sans prise en compte de la possible présence de traces archéologiques antérieures aux exploitations occidentales dans ces environnements de sols acides de montagnes. Contrairement aux espaces des vallées de la Grande Terre, où était reconnue dès la période coloniale la présence de traces kanak anciennes (Glaumont, 1888), les sites de mines ont été durant longtemps vus comme des «espaces vides », des environnements " premiers » voués à la seule exploitation industrielle. Pourtant, dès la fin de la Deuxième Guerre mondiale, des observations détaillées de vestiges archéologiques anciens avaient été publiées pour la presqu'île de Bogota à Canala, montrant la présence de cairns ainsi que d'alignements de pierres, formant parfois des murets (Avias, 1949). Plus au nord sur la côte Est, l'exploitation de la mine de Poro a nécessité dans les années 1970 le déplacement d'un ensemble de pétroglyphes, positionné sur un sommet de montagne surplombant la mer (Frimigacci, 1977).

Ce n'est qu'au cours de la dernière décennie que le patrimoine archéologique surtout préeuropéen mais également minier des plateaux latéritiques a commencé à être pris en compte de façon plus coercitive, à travers les obligations d'études d'impact imposées aux opérateurs miniers. Les prospections archéologiques (carte 1) menées dans ce cadre sur les plateaux latéritiques de Poum (Domergue et Wadrawane, 2007) et de Tiébaghi (Gony et al., 2008) dans l'extrême nord de la Grande Terre, de Koniambo (Baret et al., 2000) sur la côte Ouest et de Goro (Barp, 2006) et Ouinné (Lagarde et al., 2008) dans le sud, n'ont pas permis de mettre en évi- dence des traces claires et nombreuses de structures ou d'aménagements précoloniaux. Seuls deux sites ont pour le moment été localisés dans la zone de Goro : un alignement de murets, environné de structures en cairns sur le plateau de la plaine des lacs (Sand et Ouetcho, 1992 : fig. 51) et un abri sous roche occupé de façon sporadique à date ancienne dans la zone d'impact du barrage de l'usine de nickel de Goro (Sand, Barp et al., 2007). Les prospections menées sur une mine de Thio et dans le fond de la vallée de la Tontouta (carte 1) ont pour leur part mis en lumière la présence d'aménagements plus nombreux antérieurs au premier contact européen. Cet article présente les principaux sites inventoriés dans ces deux régions et la toute première série de datations obtenues pour ce type d'aménagements en Nouvelle-Calédonie. Ces données indiquent de façon claire que certaines zones de péridotites de la Grande Terre ont été utilisées de façon régulière ou sporadique, dans le cadre de rituels ou comme lieux de passage, bien avant leur exploitation minière.

\section{Inventaire et datations de sites pré-coloniaux de la mine SLN du Camp des Sapins (commune de Thio)}

Les opérations minières entreprises sur le plateau du Camp des Sapins (Thio) par la Société Le Nickel ont permis la découverte par les ouvriers de plusieurs structures archéologiques apparaissant anciennes. À la suite d'une demande des autorités coutumières de la tribu de Koua a été réalisé en 2007 et 2008 un premier inventaire descriptif sur différentes zones de cette mine en cours d'activité, avec le relevé en plan des structures les plus intéressantes, la réalisation d'une fouille sur un four traditionnel enterré avec pierres de chauffe et le prélèvement d'échantillons de coquillages et de charbons pour effectuer des datations au carbone 14 .

\section{La « maison du lézard» et les structures archéo- logiques environnantes}

Dans la zone de la mine Amphibole, les clans de la région indiquent un site cultuel dit « maison du lézard » (site ETH020) ${ }^{1}$, localisé en ligne de crête dans un petit creux aplani où poussent des Araucarias et où s'individualisent quatre plates-formes aménagées et des cairns en blocs de spinelle chromifère. La structure définie par la tradition orale comme "l'emplacement du

1. Les sites inventoriés ont été classés dans l'inventaire général des sites calédoniens. Les sites de Thio sont codés E pour est et TH pour Thio. Les sites de La Tontouta sont codés W pour ouest et PT pour Païta (Frimigacci et Maitre, 1981). 


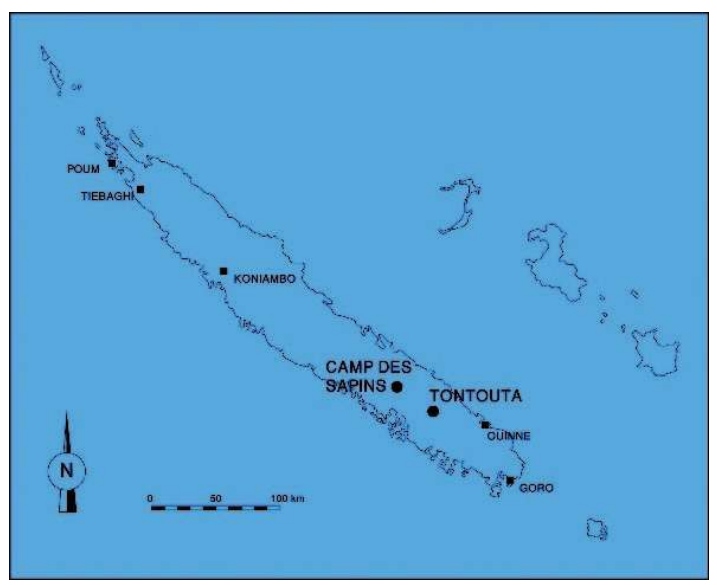

CARTE 1. - Emplacement des deux régions étudiées ici, ainsi que des différents autres points de la Grande Terre où ont été menées des prospections archéologiques sur domaines miniers

lézard » (lieu tabou) se caractérise par la présence d'un ancien ovale de blocs de chrome, de $100 \mathrm{~cm}$ de diamètre maximal, avec un reste de porte-montre (Chicoreus ramosus) à l'intérieur (photo 1). La coquille est très rongée : seules les parties les plus épaisses sont encore présentes, indiquant une relative ancienneté. L'endroit a été réaménagé récemment par des clans de Koua, avec la mise en place d'un poteau tabou sur lequel ont été fixées des étoffes. Des restes de tabac et une monnaie kanak ont également été déposés au pied du poteau. D’après une tradition orale relevée auprès de Samuel Moindou lors de la visite du site, ce lieu aurait été un ancien cimetière, bien qu'aucun reste humain n'ait été identifié lors de notre inventaire archéologique. Un squelette humain aurait été mis au jour à proximité du site ETH020 lors d'activités minières. Un fragment de coquille de Lambis sp., prélevé à l'extérieur du cercle de chrome, a été daté de 1184+/-31 BP (Wk-24279), calibré à deux sigmas $(95,4 \%$ de probabilité) entre $830 \mathrm{BP}$ et $640 \mathrm{BP}(\mathrm{BP}=$ avant 1950).

Sur la ligne de crête immédiatement au dessus du site ETH020, le site ETH019 est caractérisé par la présence de différents tas de blocs de chrome en cairns (photo 2). Les structures se trouvent sur deux mamelons séparés d'une vingtaine de mètres, avec un tumulus disposé au sommet d'un petit dôme positionné au nord et des petits amoncellements de spinelle chromifère au sud. Un autre tas est présent plus bas, en bordure de la plate-forme de mine actuelle. Des chemins anciens ont été identifiés sur la colline, l'un à côté de la «maison du lézard » (ETH020), l'autre en dessous du site ETH019. Un prélèvement d'un fragment de Lambis lambis réalisé sur un des cairns a donné une datation de 1243+/-30 BP (Wk-24280), calibrée à deux sigmas entre $890 \mathrm{BP}$ et $680 \mathrm{BP}$.

Le sommet de la ligne de crête, à environ $1000 \mathrm{~m}$ de la « maison du lézard», forme le site ETH022. Du sommet du mamelon situé à quelques mètres au-dessus d'un ensemble de structures archéologiques, on peut voir (dans le sens des aiguilles d'une montre) en partant du sud, la baie de St-Vincent au-delà de la vallée de la Ouenghi, puis les baies de la zone nord Bouloupari/Ouatom derrière le pic de Ouitchambo, les vallées de la Foa, enfin en direction de la vallée de Thio par une ouverture entre deux montagnes, le tout sans compter une vue complète sur toute la haute vallée de la Ouenghi Nord. Tout message par feu transmis à partir de ce point pouvait être vu par temps clair sur près de $50 \mathrm{~km}$ de la côte Ouest et vers la côte Est. Deux cairns sont présents en contrebas du mamelon. L'un de ces empilements comporte en son centre un bloc de péridotite de forme allongée et de section carrée, aux parois extérieures usées : il s'agit selon toute évidence d'un bloc volontairement apporté. Une coquille de portemontre (Chicoreus ramosus) prélevée à la base d'un des amas a produit une datation de 1192+/-30 BP (Wk-24277), calibrée à deux sigmas entre $840 \mathrm{BP}$ et 640 BP. À une vingtaine de mètres en contrebas de la plate-forme portant les deux cairns, dans un creux vers le sud-ouest ont été ramassés toute une série de tessons de poterie, éparpillés sur environ $5 \mathrm{~m}$ d'étendue. La poterie s'apparentant à la tradition de Néra est peu épaisse, avec un dégraissant fin. Ces vestiges archéologiques lessivés pourraient marquer l'emplacement d'un site ancien de rituels, car la zone n'est pas naturellement appropriée pour l'établissement d'un campement, se trouvant au pied d'un sommet très exposé aux vents, sans protection naturelle et loin de tout point d'eau.

Un ancien chemin (site ETH026) se dirige à partir du pied du site ETH022, le long de la pente ouest de la colline, vers les sites ETH020 et ETH021. Il semble s'agir d'un ancien «chemin kanak », probablement réutilisé par les premiers mineurs à avoir prospecté cette partie du plateau. Le site ETH032 se trouve à peu de distance au-dessus de ce chemin, sur un petit plateau naturel. La structure principale de ce site est formée d'une accumulation rectangulaire de blocs, mesurant $280 \mathrm{~cm}$ sur $270 \mathrm{~cm}$, avec une hauteur de $70 \mathrm{~cm}$ dans sa partie nord. Le remplissage est principalement composé de gros blocs de chrome, avec quelques petits galets à la base. Sept petits tas, formés en grande majorité de blocs de chrome de moins de $15 \mathrm{~cm}$ de côté, 


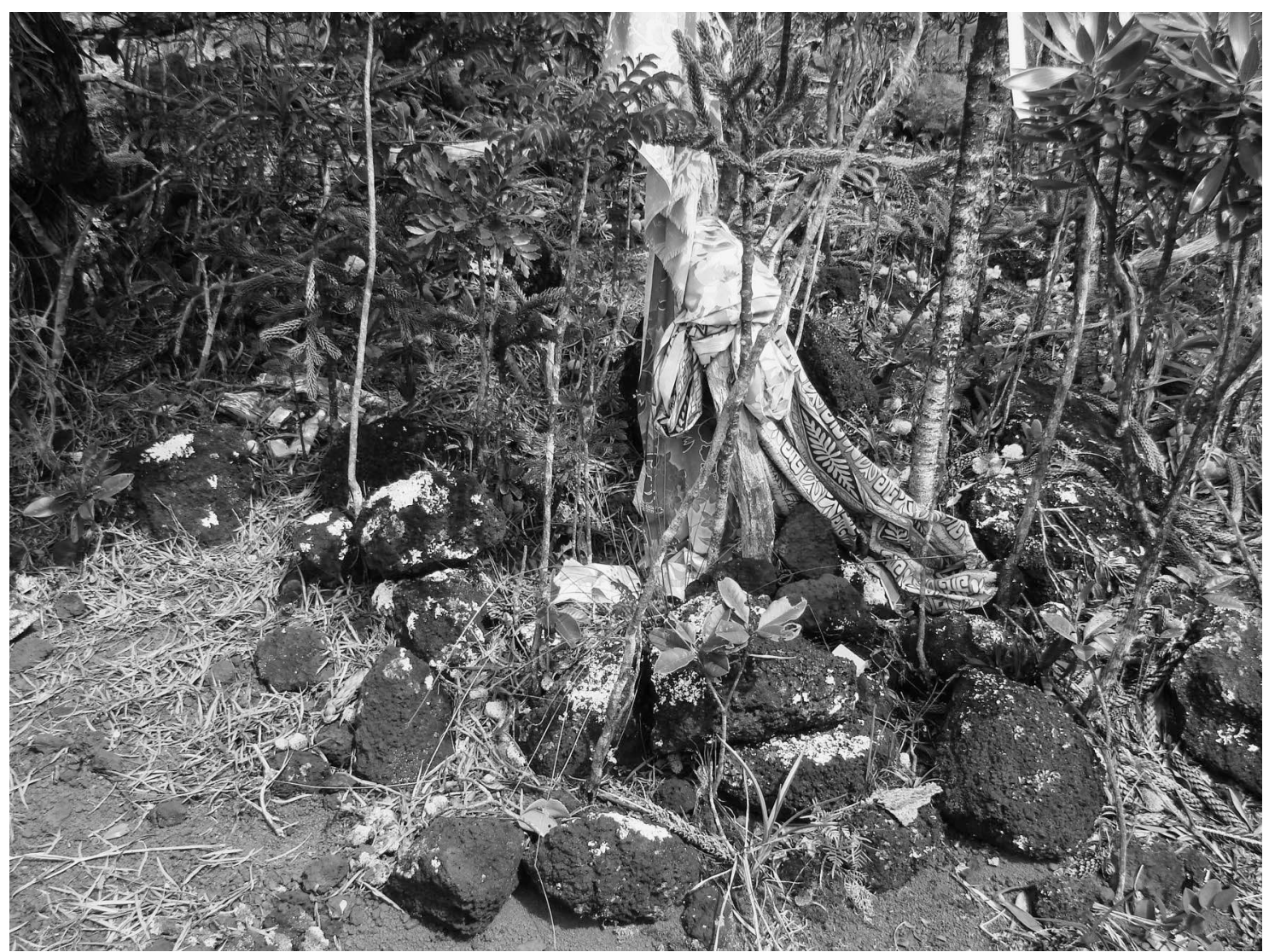

Рното 1. - Cercle de blocs de chrome marquant l'emplacement de la «maison du lézard » (ETH020), au centre duquel a été planté un poteau tabou (cliché C. Sand)

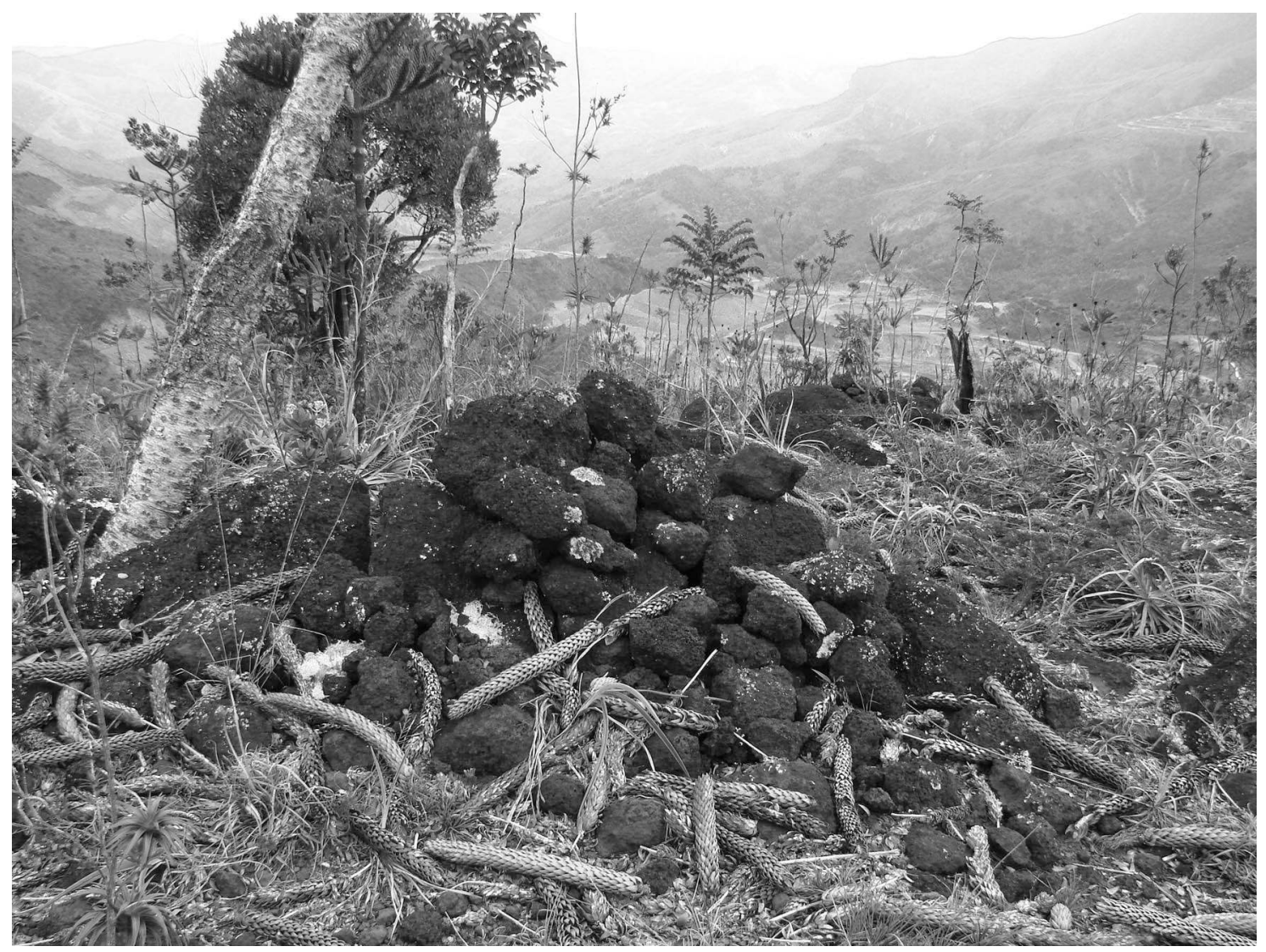

Рното 2. - Vue d'un des cairns du site ETH019 en sommet de ligne de crête. La vallée de Koua ainsi que différents plateaux de mine en cours d'exploitation se distinguent en arrière-plan (cliché C. Sand) 

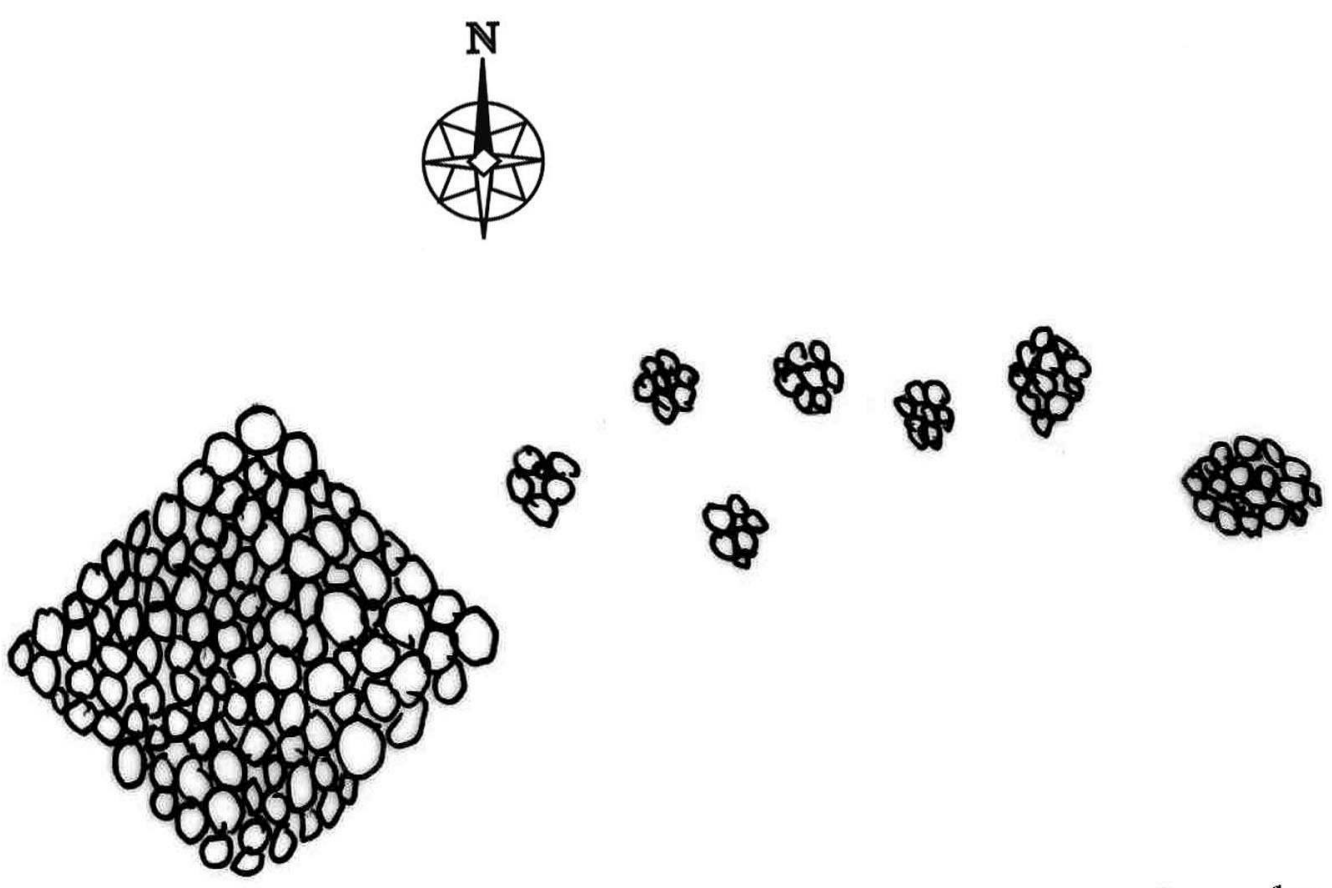

0

$1 \mathrm{~m}$

FIGURE 1. - Relevé des différents amas de blocs de chrome du site ETH032

sont alignés à partir de la structure principale. La hauteur des tas varie entre 10 et $15 \mathrm{~cm}$, avec des diamètres compris entre 55 et $80 \mathrm{~cm}$, sauf pour le plus éloigné, qui atteint $25 \mathrm{~cm}$ de hauteur et $100 \mathrm{~cm}$ de long. La succession des différents tas forme une courbe (figure 1), ce qui a priori invalide la possibilité d'un alignement pour indiquer une position éloignée sur un autre versant de montagne.

Un site plus étendu (site ETH021) a été localisé sur le versant ouvrant vers la vallée de la Ouenghi (sud-est), en contrebas immédiat de la « maison du lézard » (ETH020), au pied d'une source dans une cuvette remontant vers une bordure de colline. L'espace s'étend sur une surface d'environ $1000 \mathrm{~m}^{2}$, avec la présence de toute une série de structures aménagées visibles au sol. Sept structures de type cairn de tailles variables, en blocs de chrome, se distinguent en sommet de site. La plus importante couvre à la base environ $4 \mathrm{~m}^{2}$, avec une hauteur d'environ $100 \mathrm{~cm}$. Les autres délimitent en moyenne une base de $2,50 \mathrm{~m}^{2}$ sur $50 \mathrm{~cm}$ de hauteur. Séparé de cet ensemble qui paraît avoir été lié avant tout à des activités minières, se trouve sur la même courbe de niveau (environ $820 \mathrm{~m}$ ) un aménagement circulaire de blocs de chrome, comportant en bordure un vieux poteau de bois. Au centre du cercle a été posé un bloc de gabbro de forme allongée, environné de restes de coquillages (conque Charonia tritonis et araignée Lambis sp.) (photo 3 ). Ce site pourrait indiquer l'emplacement d'un lieu de rituels, peut-être liés aux prémices d'igname selon une tradition orale évoquée lors de l'inventaire. Un fragment de Lambis prélevé sur ce site a donné une datation de 1415+/-31 BP (Wk-24278), calibrée à deux sigmas entre 1070 BP et 840 BP.

La partie nord-ouest du site, sur la pente douce en contrebas des structures précédentes, a été aménagée à date ancienne, avec la présence d'une série de petits murets bas droits ou en arc de cercle, délimitant des plates-formes dépourvues de cailloux. Un four en pierre ainsi que des tessons d'une poterie de tradition Néra cassée sur place ont été découverts sur une de ces plates-formes. Le pot est de forme sphérique à bords rentrants, avec un diamètre moyen d'environ $35 \mathrm{~cm}$ (figure 2). Six autres emplacements avec des restes de poteries de tradition Néra ainsi qu'un autre four en cours d'érosion ont été identifiés sur le site, indiquant une occupation répétée du lieu, peut-être comme campement avant la période coloniale. La fonction des nombreux petits tas de blocs de chrome, de forme variable, localisés lors du relevé de cette partie basse du site ETH021, n'a pas été définie. 


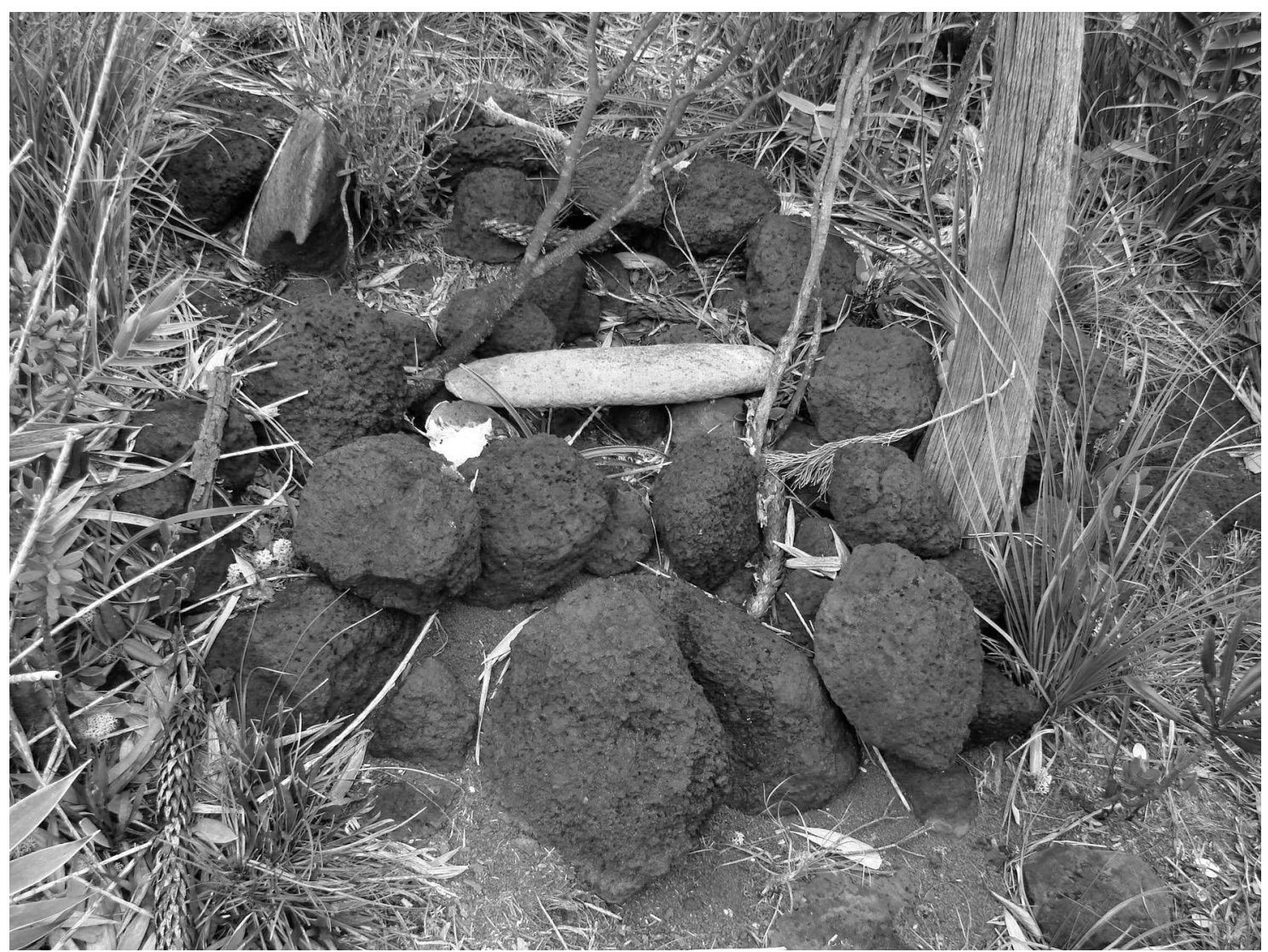

Рното 3. - Cercle de blocs de chrome, au centre duquel se distinguent une pierre allongée et des coquillages (site ETH021) (cliché C. Sand)

La présence d'un four enterré avec pierres de chauffe bien conservé sur ce site donnait l'occasion de pouvoir réaliser une étude stratigraphique de la structure de cuisson océanienne et permettait de tenter de dater sa dernière utilisation. Après le dégagement de la lentille de surface, la fouille s'est concentrée sur l'angle est du four, sur une superficie d'un quart de mètre carré. Plusieurs niveaux de pierres en superposition les uns sur les autres ont été observés. Toutes les pierres du carré ont été mesurées, la majorité étant comprise entre $5 \mathrm{~cm}$ et $10 \mathrm{~cm}$ d'extension maximale. La fouille de la couche inférieure (photo 4 ) a mis au jour beaucoup de charbons dans le sédiment sous les pierres, certains étant encore de grosse taille. Une concentration plus significative était localisée en bordure de la paroi du four. De nombreuses prises d'échantillons ont été effectuées pour identification ultérieure d'espèces.

La fouille de la base de la structure a montré la présence d'une cuvette d'environ $20 \mathrm{~cm}$ de profondeur par rapport au sol contemporain, couverte par une mince lentille de sable de chrome ocre issu probablement des lessivages des pluies. La couche du cœur du four s'enfonce

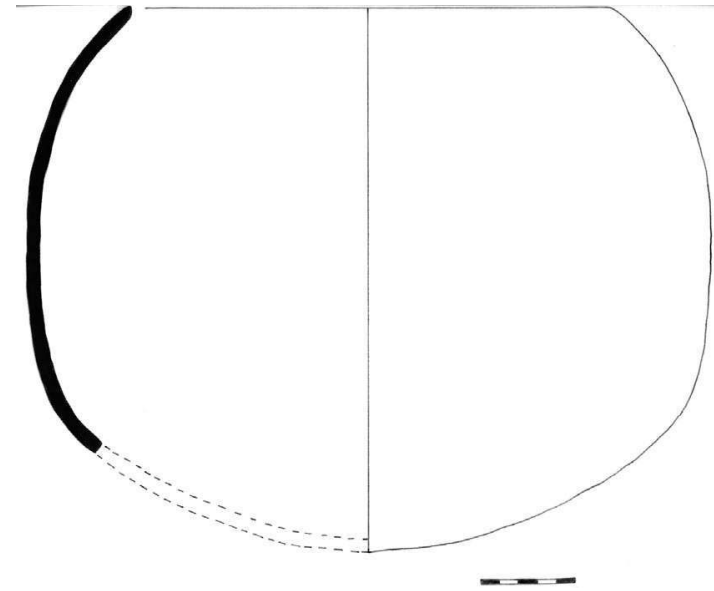

Figure 2. - Reconstitution d'une poterie de la tradition de Néra découverte sur le site ETH021 (dessin A.-J. Ouetcho)

clairement en dessous de la limite inférieure des pierres les plus profondes. Les charbons sont présents jusqu'à la base de la cuvette, certains étant posés à plat au fond. Cette configuration pose la question de l'utilisation répétée du four au cours du temps. La bonne tenue des charbons dans un sol acide est une indication d'une faible 


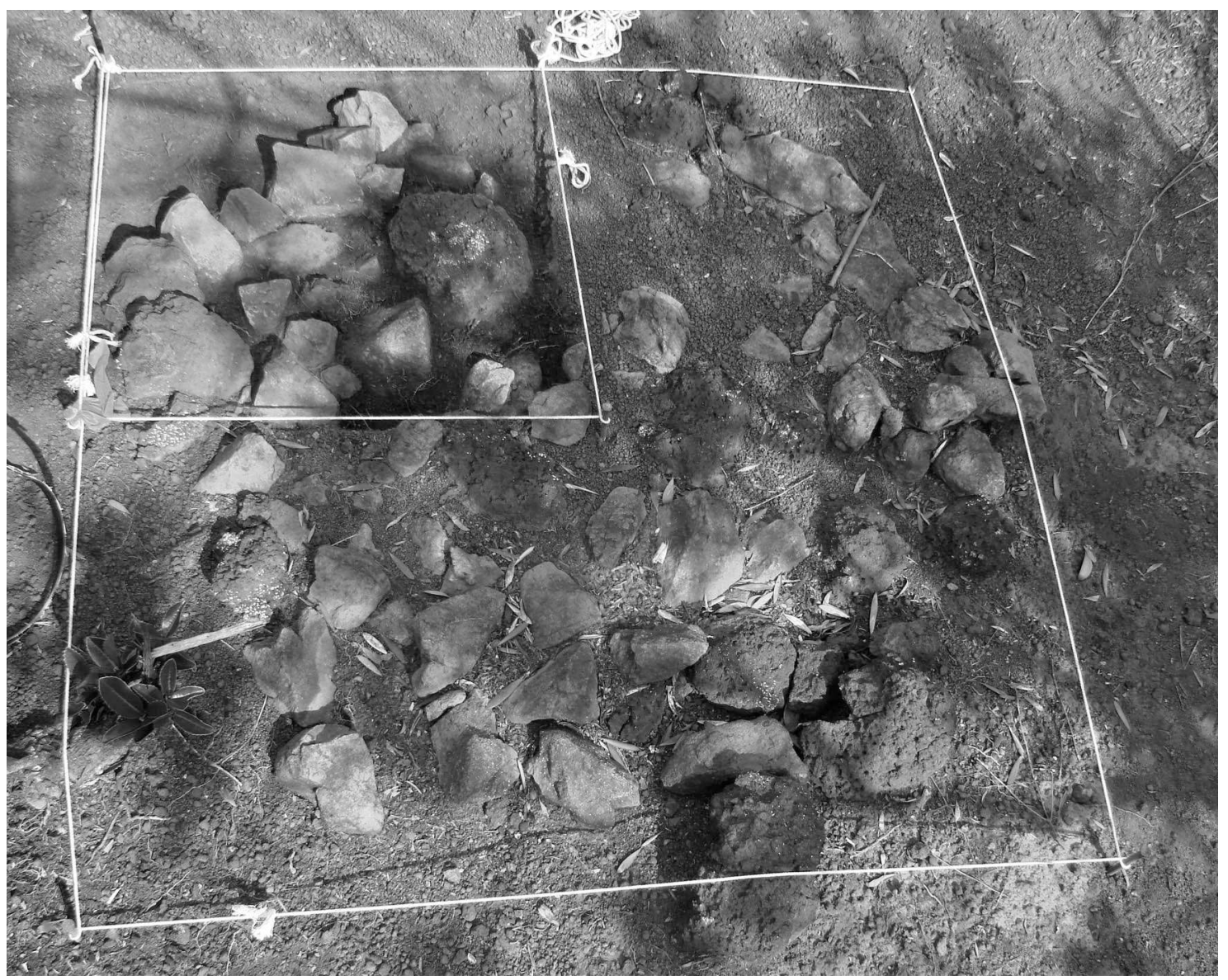

Рното 4. - Four étudié sur une des plates-formes du site ETH021. On observe un « effet de paroi » en bordure de l'ange fouillé de la structure (cliché C. Sand)

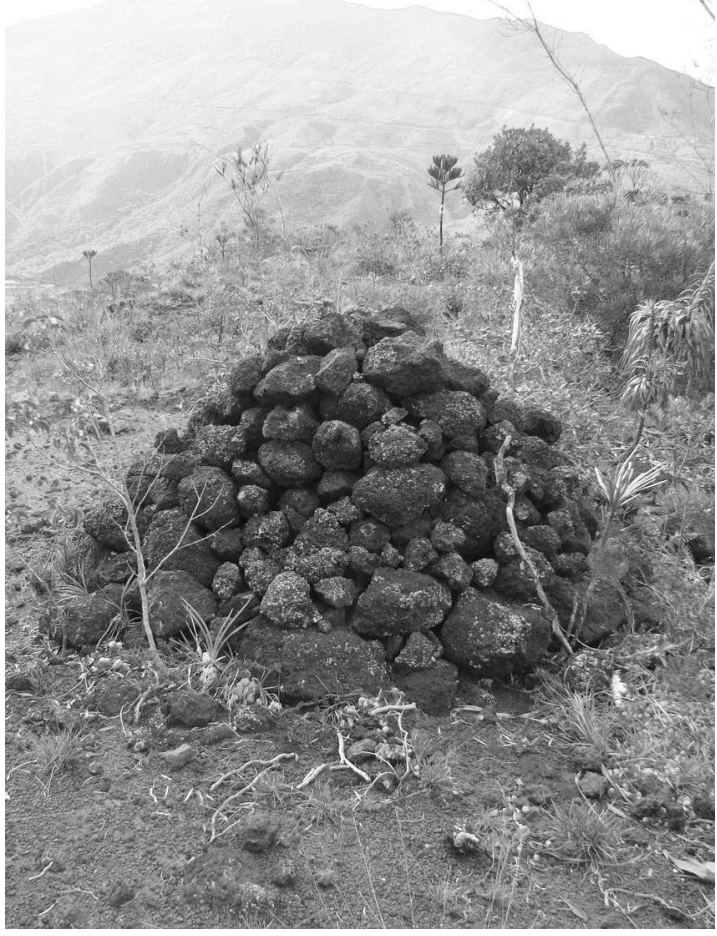

Pното 5. - Grand cairn du site ETH023 (cliché C. Sand) ancienneté de la dernière utilisation. Toute une série de charbons a été prélevée pour possible datation ${ }^{14} \mathrm{C}$, de la zone des pierres jusqu'au fond, afin de tenter d'identifier des différences de datations. Deux de ces échantillons ont donné respectivement une datation de 175+/-30 BP (Wk-24282), calibrée à deux sigmas entre $290 \mathrm{BP}$ et le présent et de 142+/-32 BP(Wk-24283), calibrée à deux sigmas entre $270 \mathrm{BP}$ et le présent. Ces deux dates comportent chacune une première plage de calibration, respectivement entre $280 \mathrm{BP}$ et $210 \mathrm{BP}$ pour Wk-24282 et entre $260 \mathrm{BP}$ et $210 \mathrm{BP}$ pour $\mathrm{Wk}-24283$, soit la deuxième moitié du XVIII ${ }^{\mathrm{e}}$ siècle.

Autres sites archéologiques anciens de la mine du Camp des Sapins

Localisé à environ $700 \mathrm{~m}$ de la «maison du lézard » vers l'ouest/sud-ouest, le site archéologique ETH023 est disposé sur le versant de la longue vallée menant à la tribu de Koua. Cet aménagement positionné sur la pente d'une colline comprend une série de cairns et un tumulus 


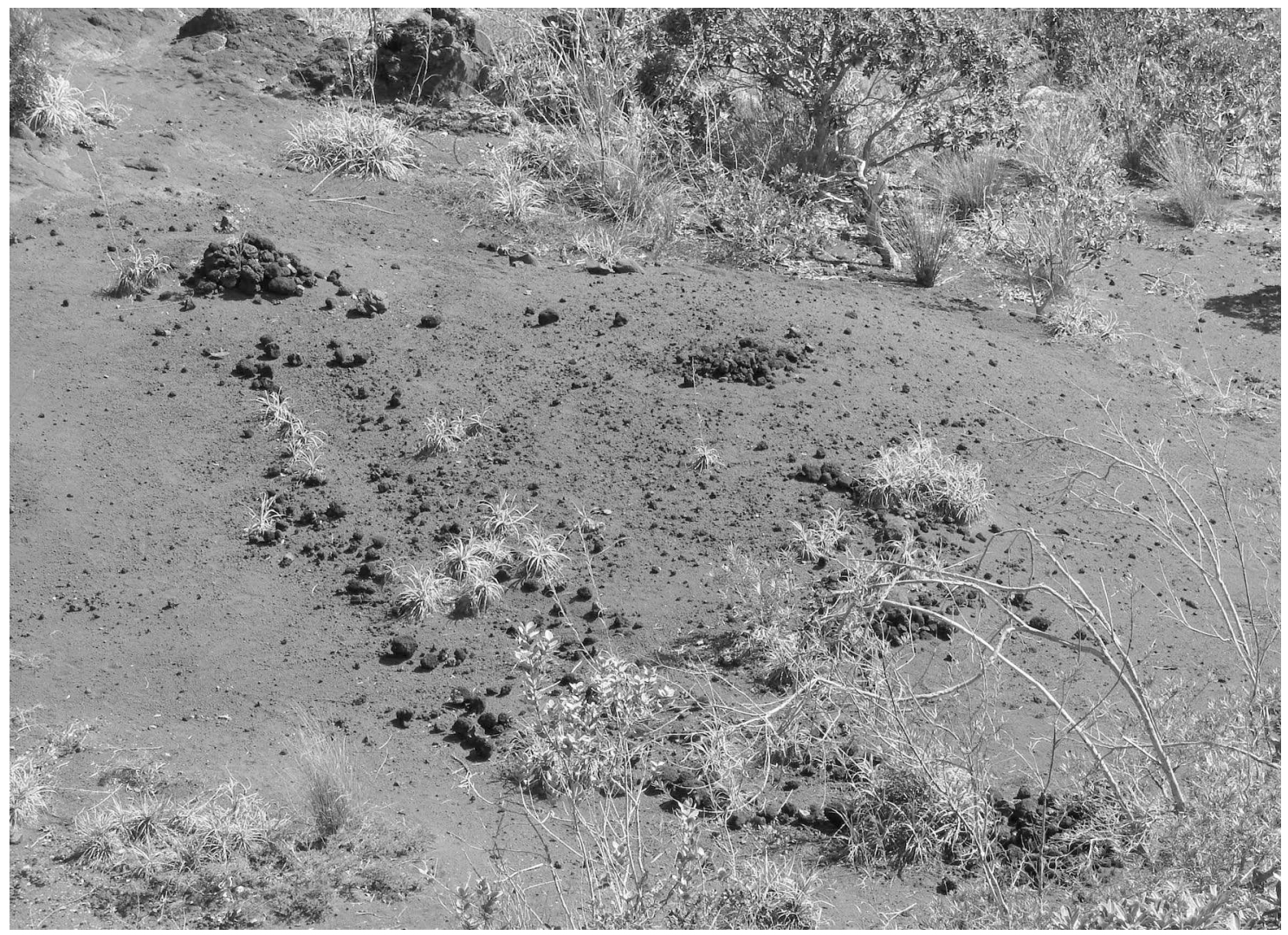

Рното 6. - Vue générale du double alignement et de différents amas de blocs de chrome du site ETH038 (cliché C. Sand)

allongé rectangulaire. Le cairn principal, de forme arrondie, d'environ $240 \mathrm{~cm}$ de diamètre à la base et $100 \mathrm{~cm}$ de hauteur, renferme des fragments de coquillages (photo 5), dont une porcelaine blanche Ovula ovum, laissée en place. Un fragment de conque Charonia tritonis (Cymatiidae) a été prélevé et daté de 467+/-30 BP (Wk24281), calibrée à deux sigma entre $231 \mathrm{BP}$ et le présent. Le tumulus rectangulaire présent à proximité, mesurant $200 \mathrm{~cm}$ de long sur $60 \mathrm{~cm}$ de large et $50 \mathrm{~cm}$ de hauteur moyenne, semble trop étroit pour avoir été une tombe. En contrebas de ces structures se trouvent différentes petites plates-formes en blocs de chrome et d'autres cairns, qui apparaissent liés à des prospections minières.

Lors de la prospection de la zone de la mine Cerise/Crépuscule, localisée en surplomb de la vallée menant à la tribu de Koua, sur la dernière zone de péridotites avant les ensembles géologiques sédimentaires, deux sites archéologiques ont été découverts. Le premier (site ETH037) est caractérisé par une concentration de tessons de poterie au sol sur un terre-plein peu rocheux localisé juste avant le tombant de falaise menant à la rivière, $250 \mathrm{~m}$ en contrebas. Les fragments de poterie, dispersés sur environ $3 \mathrm{~m}^{2}$ entre des blocs de péridotite, ont été brisés en des dizaines de morceaux par le passage répété des cerfs et des cochons : certains tessons se trouvaient même enfouis sous la couche d'humus.

Sur un autre bout de promontoire au sudouest du site ETH037, en bordure d'un chemin ancien menant probablement vers le bas de la vallée, a été découvert un aménagement comprenant des cairns de différentes tailles et un double alignement de blocs de chrome (ETH038). L'orientation générale est est/ouest (photo 6). La structure centrale est limitée par deux gros cairns. Celui à l'ouest mesure à sa base environ $100 \mathrm{~cm}$ sur $80 \mathrm{~cm}$, avec une hauteur moyenne conservée de $30 \mathrm{~cm}$. Un bloc de péridotite aujourd'hui éboulé avait été placé à date ancienne au sommet de la structure. Le tas à l'est mesure $110 \mathrm{~cm}$ sur $100 \mathrm{~cm}$ à sa base et a une hauteur de $40 \mathrm{~cm}$. Un bloc de péridotite est encore présent à son sommet. Ces deux cairns sont séparés par une distance de $11,8 \mathrm{~m}$ et reliés par une sorte de "chemin » de forme courbe orienté sud/sud-est, d'une largeur extérieure moyenne d'environ $110 \mathrm{~cm}$. Trois petits cairns, d'une dizaine de centimètres de hauteur chacun, s'alignent sur le bord nord/nord-ouest de ce «chemin ». Le premier à partir de l'est a une 


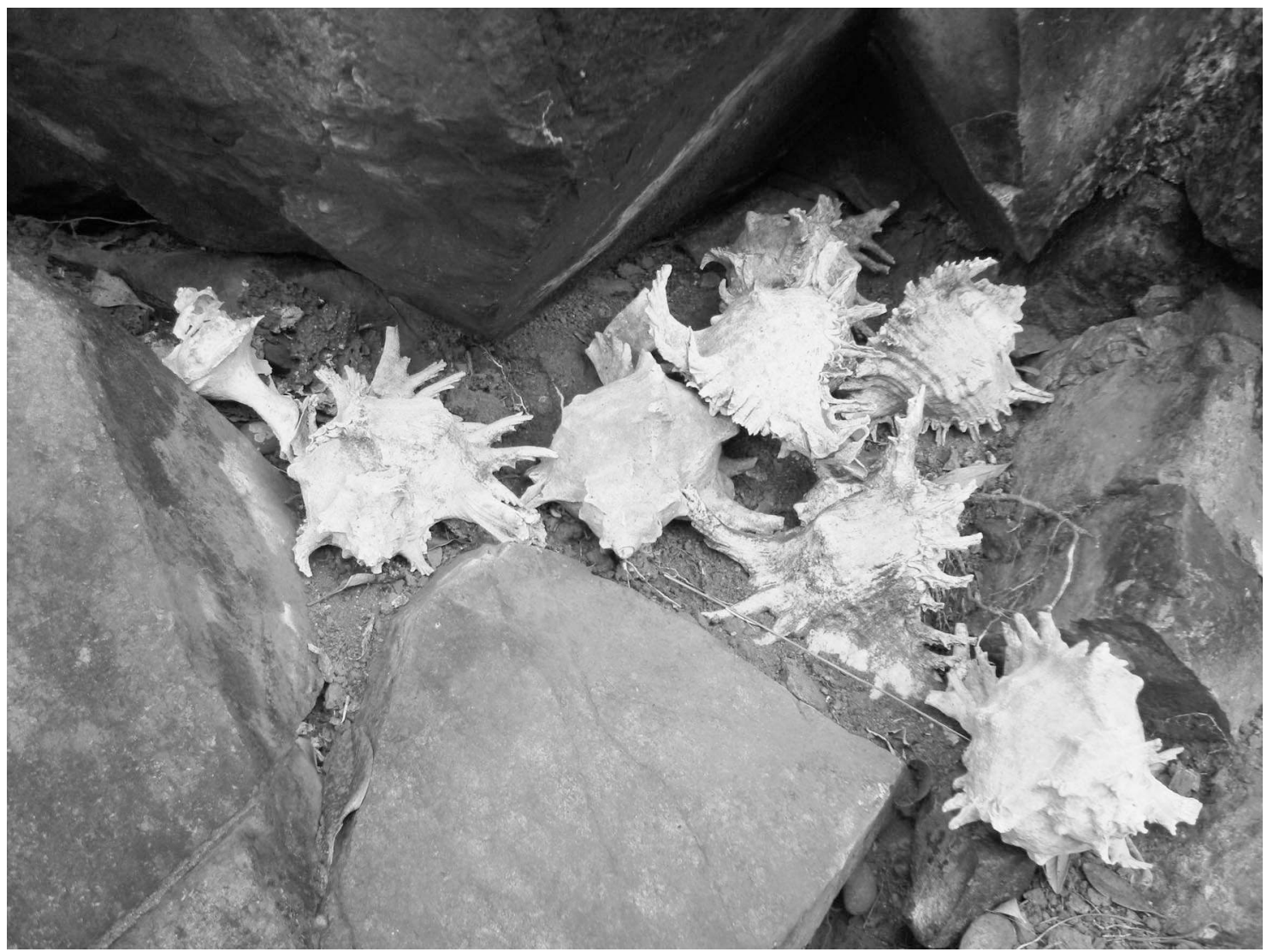

Рното 7. - Concentration de coquilles de Murcidae placées sur une plate-forme naturelle de la haute Tontouta (site WPT130) (cliché C. Sand)

assise à la base de $50 \mathrm{~cm}$ sur $60 \mathrm{~cm}$, avec un bloc de péridotite sur son sommet. Le tas central, aujourd'hui partiellement éboulé, a un diamètre général de $70 \mathrm{~cm}$ et comporte également un bloc de péridotite sur son sommet. Le troisième présente un diamètre moyen de $80 \mathrm{~cm}$. Un autre petit cairn isolé est présent à environ $4 \mathrm{~m}$ vers le nord-ouest cet ensemble. Il a un diamètre de $70 \mathrm{~cm}$ et une hauteur de $25 \mathrm{~cm}$.

Une structure (site ETH027) de même configuration générale a été découverte sur le versant opposé de la mine du Camp des Sapins, à environ $1000 \mathrm{~m}$ à l'est du sommet du site ETH022, au niveau de la ligne de partage des eaux surplombant la mine de Sapins-Sud. Cet aménagement comprend trois petits amas de blocs de pierres côté sud/sud-est et un amas au nord-ouest, séparés par un espace d'environ $7 \mathrm{~m}$ de longueur délimité par un double alignement de rognons de chrome. Le tout a été construit sur le sommet plat d'une petite ligne de crête. Aucun autre aménagement évident n'a été observé aux alentours immédiats.

\section{Vestiges pré-coloniaux de la vallée de la rivière du Humboldt (haute Tontouta, commune de Païta)}

Les nombreux affluents de la vallée de la Tontouta ont depuis la fin $\mathrm{du} \mathrm{XIX}^{\mathrm{e}}$ siècle été le cadre de prospections minières intensives. Deux grandes mines fonctionnent encore de façon permanente, l'une au pied du Mont Humboldt (mine Vulcain) et l'autre près du déboucher de la vallée alluviale (mine Sombeba). Une prospection menée dans la zone moyenne de la vallée de la rivière du Humboldt, qui forme une des vallées aboutissant à la ligne de partage des eaux au nord de la haute Kalouéhola, a permis à M. Jacquet de localiser différentes traces d'occupations pré-coloniales dans un vallon surplombé par d'anciennes prospections minières.

$\mathrm{Au}$ pied de la falaise marquant le déboucher d'un petit creek, se trouve le site WPT130, caractérisé par une concentration de grosses coquilles de Murcidae. En suivant le cours des rivières, l'accès au bord de mer se trouve à plus de $30 \mathrm{~km}$ de ce point. La concentration de ces coquilles de grande taille et connues pour avoir une importance dans la symbolique des sociétés de Méla- 
nésie, identifie ce site comme un possible lieu de rituels, ou du moins un lieu de passage au pied duquel étaient laissés des coquillages symboliques. Certaines coquilles semblent plus anciennes que d'autres (photo 7). Un Chicoreus ramosus a été prélevé pour datation et a donné un résultat de 1313+/-32 BP (Wk-25257), calibré à deux sigmas après corrections entre $940 \mathrm{BP}$ et $760 \mathrm{BP}$.

Sur la rive gauche en remontant le creek, ont été identifiés des aménagements de terrasses rudimentaires qui pourraient avoir été des plates-formes pour des campements (site WPT131). En effet, ce point permet de débuter l'ascension de la face sud du Humboldt par un ancien chemin kanak et d'atteindre un col d'accès à la côte Est. À l'arrière de ces aménagements rudimentaires, il y a une dizaine d'années, a été découverte dans une anfractuosité une poterie à anses presque complète, brisée en une dizaine de gros fragments (photo 8). Celle-ci a une forme en obus, une hauteur de $60 \mathrm{~cm}$ et un diamètre maximal de $35 \mathrm{~cm}$. Le diamètre de l'ouverture est de $21 \mathrm{~cm}$ et le bord est rentrant.

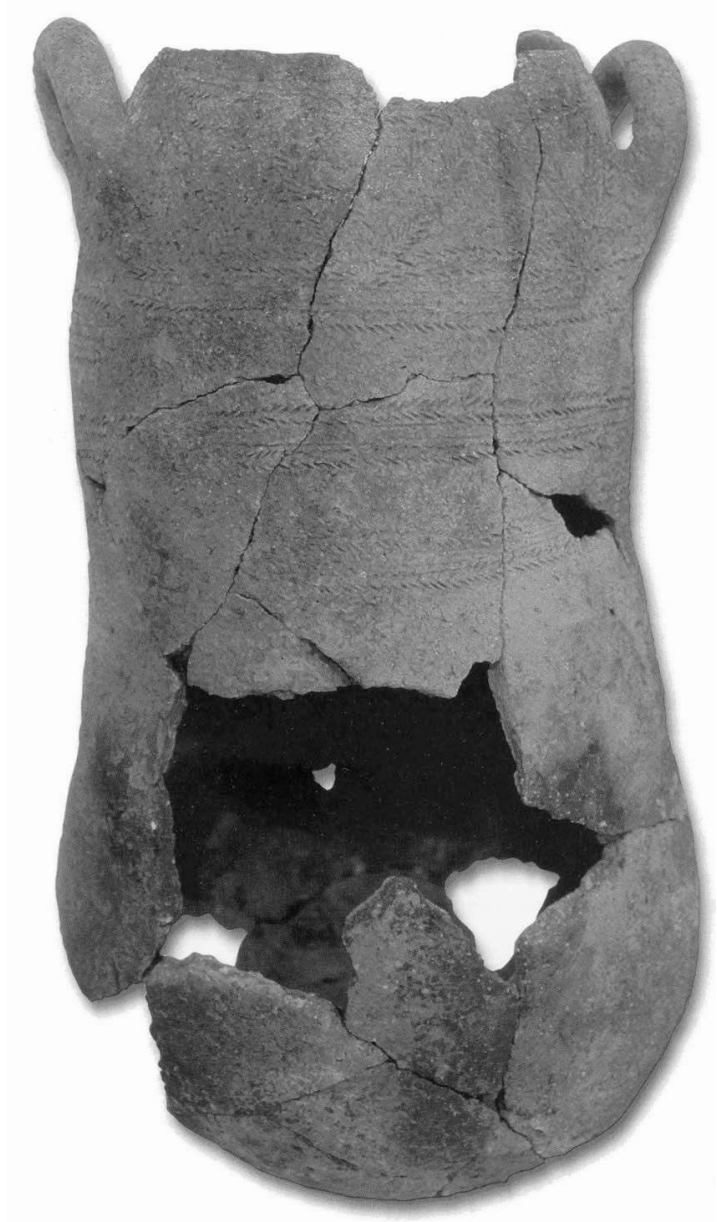

Рното 8. - Poterie à anses de la tradition de Plum du site WPT131 après restauration (cliché C. Sand)
Les anses, placées sous le bord, sont de section ronde. La partie supérieure du récipient est décorée de motifs incisés en palme, une décoration classique des poteries de la tradition de Plum fabriquées durant le premier millénaire après J.-C. dans le sud de la Grande Terre (Chevalier, 1966-70; Sand et al., 2011). Une coquille de conque Charonia tritonis (Cymatiidae), prélevée à proximité de l'anfractuosité contenant la poterie, a donné une datation de 1696+/-30 BP (Wk25258), calibrée à deux sigmas après corrections entre $1320 \mathrm{BP}$ et $1170 \mathrm{BP}$. En circulant dans la haute vallée de la rivière, se distinguent dans le paysage toute une série de chemins probablement pré-européens.

\section{Analyse}

Les données archéologiques issues de l'inventaire partiel du plateau minier de la SLN autour du Camp des Sapins et d'un vallon de la rivière du Humboldt dans la haute Tontouta indiquent la présence de vestiges culturels anciens, en parallèle à des structures associées aux activités minières (Sand et al., 2008a). Les premiers, formés principalement de cairns, d'alignements de pierres, de plates-formes et d'indices de passages matérialisés par des fragments de poteries, des coquillages et des fours en pierre, sont datables en majorité de la période pré-coloniale. Les autres, composés en grande partie de sondages de prospections minières, sont des traces liées aux puits de minerai. Il est apparu que ces deux ensembles de vestiges se trouvaient régulièrement à proximité l'un de l'autre. Ceci est probablement dû avant tout à l'emprunt, à partir de la deuxième moitié $\mathrm{du} \mathrm{XIX}^{\mathrm{e}}$ siècle, des chemins kanak anciens de passage entre les deux côtes par les premiers prospecteurs européens, selon toute vraisemblance guidés par des pisteurs kanak de la région.

Le pot à anses complet découvert dans la haute Tontouta est clairement de la tradition de Plum, datée du premier millénaire après J.-C. Les tessons de poterie découverts au cours de l'inventaire du Camp des Sapins se rattachent tous à la tradition de Néra, développée dans le sud de la Grande Terre au cours du deuxième millénaire après J.-C. (Sand et al., 2011). La présence de cette tradition céramique bien datée vient en complément des résultats des datations au carbone $14\left({ }^{14} \mathrm{C}\right)$ sur des coquillages associés à plusieurs structures archéologiques, pour indiquer une circulation sur le plateau de Thio principalement durant le deuxième millénaire après J.-C., alors que les passages apparaissent débuter 
plus anciennement dans la haute Tontouta. En effet, l'analyse des résultats de la première série de datations ${ }^{14} \mathrm{C}$ réalisée à ce jour sur ce type de site archéologique calédonien permet d'identifier trois ensembles chronologiques distincts.

Le premier, limité à la haute Tontouta, montre des passages au cours de la seconde moitié du premier millénaire après J.-C., ce qui correspond bien à l'époque de production de la tradition à anses dans le sud de la Grande Terre. Durant cette période a été identifiée une situation de crises à travers l'archipel calédonien, en partie due à un impact trop marqué sur l'environnement insulaire, lié à une multiplication des brûlis dans le cadre de traditions horticoles simples, réalisés par une population en augmentation démographique (Sand et al., 2008b). Il est possible que les tensions aient incité certains groupes à tenter des implantations dans des régions marginales de la Grande Terre, en particulier en expérimentant les possibilités horticoles des sols latéritiques.

Le deuxième ensemble chronologique, regroupant la majorité des datations obtenues sur des coquillages réalisées sur différents sites de la mine du Camp des Sapins, se place durant la première moitié du deuxième millénaire après J.-C. Cette période se caractérise plus généralement dans la chronologie archéologique calédonienne par le développement de « l'ensemble culturel traditionnel kanak», avec la création de nouveaux réseaux d'alliances et une sédentarisation des groupes claniques, qui ont engendré la mise en place de nouveaux rituels et de sites sacrés associés (Sand et al., 2003). Il est probable que les passages sur le plateau de Thio à cette époque aient été liés à des « chemins d'alliance " entre, d'une part, les plaines de la Tontouta et de la Ouenghi et, d'autre part, la vallée de Thio. Différentes marques sous forme de tas apparaissent avoir été aménagées le long des parcours, en relation dans certains cas avec des rituels.

Le troisième ensemble comprend les trois datations sur charbon et coquillage, toutes calibrées des derniers siècles. Elles sont probablement liées aux déplacements des groupes consécutifs aux premiers contacts avec les Européens et à la déstructuration graduelle des systèmes traditionnels kanak anciens qui s'en est suivie. Ce bouleversement a été causé en particulier par l'introduction de maladies nouvelles, qui ont engendré des épidémies et ont poussé de nombreux groupes à quitter leurs terroirs anciens (Sand, Bolé et Ouetcho, 2007). Toute une série de traditions orales dans la région parle de ces déplacements, qui ont par exemple entraîné des groupes à travers la montagne, de la Ouenghi sur la côte Ouest jusqu'à Borindi sur la côte Est (Guiart, 1963), en suivant des chemins préétablis entre les deux côtes. Un de ces passages semble être marqué par un four dans le campement ETH021 et peut-être de fragments de poteries de la tradition de Néra, à proximité immédiate d'une source d'eau douce qui devait être connue de longue date. L'absence de vestiges métalliques à proximité du four et sur les plates-formes du site, corrélé à la présence de poterie, invalide l'hypothèse d'un passage à la fin du XIX ${ }^{\mathrm{e}}$ siècle car, à l'époque, les poteries traditionnelles kanak avaient été remplacées par des marmites en fonte dans le sud de la Grande Terre. La sépulture découverte lors des activités minières en bordure de la zone est peut-être associée à la même phase historique de déplacements entre les côtes Ouest et Est. En effet, diverses observations réalisées lors de prospections archéologiques dans d'autres régions latéritiques du sud de la Grande Terre ont montré que l'acidité des sols miniers ne permet pas la conservation d'ossements sur plus de quelques centaines d'années.

La tradition liée à cette voie de passage entre les deux côtes a perduré durant la période coloniale, puisque le dernier passage remémoré sur ce chemin kanak remonterait à la fin des années 1940, pour un mariage. Il est donc relativement logique de penser que les premiers prospecteurs aient également emprunté les « chemins kanak » lors des premières ascensions vers le massif au cours de la deuxième moitié du XIX ${ }^{\mathrm{e}}$ siècle, selon toute probabilité accompagnés par des guides kanak. La présence de zones de prospection en surplomb immédiat du site de campement ETH021 et en contrebas des cairns du site ETH023 du Camp des Sapins n'a dans ce contexte rien d'exceptionnel et n'est probablement pas due au hasard. Une hypothèse similaire peut être avancée pour les premières prospections dans la haute Tontouta.

Si l'époque de construction des cairns et des structures découvertes sur différents plateaux miniers peut pour la première fois être datée avec une certaine précision, l'utilisation de ces aménagements est en revanche difficile à définir en l'absence de données précises des traditions orales. L'exemple du site ETH020, décrit comme la « maison du lézard » par les informateurs de la tribu de Koua, montre combien l'utilisation des sites d'anciens rituels ou liés à des clans ne peut être définie qu'à partir des connaissances transmises par les descendants des anciens utilisateurs. Les structures découvertes sur la zone du Camp des Sapins se rapprochent d'autres ensembles signalés par exemple sur la presqu'île de Bogota dans la région de Canala dès la fin des 
années 1940 par le géologue Jacques Avias et publiés dans différents articles (Avias, 1949, 1953). Les interprétations proposées à l'époque s'articulaient autour de l'idée que ces traces étaient les « vestiges d'une civilisation différente de la civilisation indigène actuelle » (Avias, 1949 : 44), une hypothèse que les datations obtenues à Thio et dans la haute Tontouta permettent de définitivement réfuter.

Des informations sur des structures se rapprochant de celles décrites dans le présent article sont également mentionnées dans la littérature ethnographique calédonienne et permettent de donner un éclairage sur l'utilisation possible de certains de ces aménagements. Le pasteur Maurice Leenhardt, travaillant principalement dans la région de Houaïlou, en bordure des massifs de péridotites du centre de la Grande Terre, a ainsi publié quelques données de traditions orales sur des amas de pierres. L'ethnologue notait que :

«dans les régions montagneuses, on trouve des monticules de pierres [...] disposés avec ordre et atteign[an]t parfois de très grandes dimensions. Ces monticules représentent des tas d'ignames élevés lors des pilous, ils sont le simulacre de l'abondance des vivres. Lorsqu'on désirait avoir un pilou, on dressait ces tas sur la montagne et l'on priait ensuite celle-ci, ses totems et ses dieux, de favoriser les récoltes et d'assurer ainsi au prochain pilou en l'honneur des ancêtres, richesse et plénitude des vivres. [...] Les plus remarquables de tous ces tas se trouvent dans un endroit perdu de la montagne de Goeweou, à $200 \mathrm{~m}$ au-dessus du niveau du centre européen de Houailou. Les indigènes ont réalisé là une représentation complète du pilou. À cet endroit peu accessible, ils ont utilisé les crêtes qui séparent les ravinements pour figurer des allées, les boeweye, et ils ont disposé tout le long des lignes des tas de pierres représentant les parts distribuées à chaque clan. Au-dessus, au point où convergent ces allées, ces crêtes, un soutènement rappelle le tertre de case : des pierres, le foyer, l'autel du pilou, et deux grosses pierres représentant des rhea. » (Leenhardt, 1937 : 241-242)

Une autre tradition, moins détaillée, a été relevée pour un site de rituels sur la presqu'île de Poum (nord de la Grande Terre) avant l'ouverture d'une mine. Le seul site identifié sur la montagne lors de l'inventaire de la presqu'île, localisé à proximité du sommet (site de Paevala, NPO17), marquait :

« le point de rencontre des différents clans de la presqu'île avant qu'ils ne se dirigent vers la zone d'échanges de la doline. D'après certains témoignages, l'espace était jusqu'à une époque récente matérialisé par un alignement de pierres formant un arc de cercle. Situé sur le plateau, cet endroit est connu de la tradition orale comme étant le lieu d'échange de denrées alimentaires entre les clans Boaouva, Padi et Boula. » (Domergue et Wadrawane, 2007 : 27)

\section{Conclusion}

Le renouvellement d'études archéologiques sur les plateaux miniers calédoniens confirme le caractère original des sites anciens présents dans des milieux géologiques particuliers comme les zones à péridotites. Il apparaît clair que les sites liés aux premiers temps de l'activité minière et aux prospections géologiques des $\mathrm{XIX}^{\mathrm{e}}$ et $\mathrm{XX}^{\mathrm{e}}$ siècles, caractérisés par de petites platesformes rectangulaires et des puits de sondages, sont les plus facilement identifiables (Sand et al., 2008b). Cependant divers cairns, alignements de blocs de chrome, structures arrondies, platesformes aménagées et dépôts de coquillages ou de poteries présents sont plus anciens, démontrant une utilisation pré-européenne d'une partie de ces plateaux de montagne aux sols acides. Leur présence pousse également à questionner l'absence actuelle de sites de même type sur d'autres massifs, qui ont pu être détruits ou lessivés au cours $\mathrm{du} \mathrm{xx}^{\mathrm{e}}$ siècle par des activités minières à l'impact plus important.

Il est probable qu'un travail de collecte de traditions orales kanak et européennes sur ces aménagements des plateaux miniers pourrait encore apporter des précisions sur les utilisations et pratiques anciennes liées à certains ensembles de structures. De toute évidence, les structures pré-coloniales de la mine du Camp des Sapins par exemple ne peuvent être séparées des traces d'occupation kanak anciennes qui s'observent en particulier jusqu'au pied du massif minier dans la vallée de Koua, montrant la présence de vestiges de terrasses de tarodières, de tertres de cases et de différents sites de pétroglyphes le long de la rivière (Ouetcho, 2008). Mais réduire toute structure hors enclos de calibrage de sols de mine à des aménagements kanak est probablement une erreur. La possibilité que certaines structures telles celles du site ETH024 du Camps des Sapins seraient des anciens repères de géomètre doit également être considérée.

Un autre aspect de l'archéologie des plateaux miniers, combinant période traditionnelle kanak et premières explorations minières coloniales, concerne les chemins d'accès encore marqués au sol. Les premiers prospecteurs ont certainement emprunté des chemins qui existaient déjà et qui leur ont été probablement souvent montrés par les guides kanak eux-mêmes. Il serait intéressant de réaliser une cartographie de ces anciens chemins, afin de définir leur répartition entre les lieux aménagés, les vallées et les plateaux. Ce travail serait également susceptible de permettre la découverte de nouveaux sites. 
Les programmes archéologiques réalisés sur différents plateaux miniers démontrent l'existence d'un réel potentiel historique et archéologique. On ne peut dès lors que saluer l'initiative des opérateurs miniers calédoniens, des autorités coutumières kanak et des responsables culturels provinciaux qui ont mis en place des études et inventaires de ce patrimoine original. Ces résultats viennent également nourrir le débat très actuel sur les droits et éventuelles compensations financières que les populations locales peuvent réclamer aux opérateurs miniers (Brutti, 2007), dans le cadre de stratégies de revendications politiques bien comprises et où l'archéologie devient souvent un argument utilisé à des fins extrascientifiques (Bradshaw, 2000). Tout en gardant en perspective ces données sociétales (Le Meur, 2010) dans lesquelles doit s'insérer l'action des archéologues contemporains, on ne peut qu'émettre le souhait que ce type de travail puisse contribuer à permettre le développement des opérations minières, auxquelles sont de plus en plus associées les populations locales océaniennes, tout en prenant en compte le patrimoine culturel et l'environnement des pays concernés.

\section{Remerciements}

Les études archéologiques présentées dans cet article ont été autorisées par la province Sud de la Nouvelle-Calédonie. La Société Le Nickel, par la voix de son ancien chef d'exploitation à Thio, M. Jouband, faisant suite à une demande des autorités coutumières de la région (tribu de Koua, au pied du plateau minier) par l'intermédiaire de M. Samuel Moindou, a souhaité que soit réalisé l'inventaire des zones en cours d'exploitation ou prochainement impactées par des projets miniers sur la mine du Camp des Sapins. Les sites de la haute vallée de Tontouta ont été découverts par M. Charles Jacquet qui a hébergé C. Sand à son campement lors de l'inventaire préliminaire. Tous nos remerciements vont aussi aux différentes personnes qui sont intervenues lors de ces programmes.

\section{BIBLIOGRAPHIE}

Avias Jacques, 1949. Contribution à la préhistoire de l'Océanie: les tumuli des plateaux de fer en Nouvelle-Calédonie, Journal de la Société des Océanistes 5, pp. 15-50.

—, 1953. La préhistoire néo-calédonienne, Journal de la Société des Océanistes 9, pp. 55-63.
BAREt David, Jacques Bolé, André-John Ouetcho et Christophe SAND, 2000. Étude de potentiel et préinventaire des ressources patrimoniales du milieu, Nouméa, Projet Koniambo, études environnementales de base.

BARP Frédéric, 2006. Usine du Sud - Projet Goro Nickel. Inventaire raisonné des sites archéologiques de surface, Nouméa, Rapport Association océanienne d'Archéologie.

BRADSHAw Elizabeth, 2000. Mining and cultural heritage managment: the Hamersley Iron experience, in Ian Lilley (ed.), Native Title and the Transformation of Archaeology in the Postcolonial World, Sydney, Oceania Monographs 50, pp. 10-23.

BRUTTI Lorenzo, 2007. From fertility rituals to mining companies: Ecocultural issues and the land rights in Oksapimin, Journal de la Société des Océanistes 125, pp. 249-255.

Chevalier Luc, 1966-1970. Les éléments de préhension de la poterie calédonienne, Études mélanésiennes 21-25, pp. 45-54.

DOMERGUE Stéphanie et Jean-Marie Wadrawane, 2007. Étude de potentiel archéologique de la presqu'île de Poum, Nouméa, "Projet POUM », Groupe SLN-ERAMET.

FrimigaCCI Daniel, 1977. Rapport sur le site pétroglyphique de Mélé (Poro), Nouméa, Centre orstom.

Frimigacci Daniel et Jean-Pierre Maitre, 1980. Code pour désigner les sites archéologiques de NouvelleCalédonie et Dépendances, Journal de la Société des Océanistes XXXVI, 66-67, pp. 125-127.

Glaumont Gustave, 1888. Étude sur les us, mœurs, coutumes, funérailles des Néo-Calédoniens. Théorie du continent englouti. Origine des Néo-Calédoniens, Nouméa, document lithographié.

Gony Yves-Béalo, André-John Ouetcho, Jacques BoLÉ et David BARET, 2008. Rapport d'intervention archéologique sur le site minier de Tiébaghi (Koumac) - Phase 2, Nouméa, rapport du département Archéologie du service des Musées et du Patrimoine de Nouvelle-Calédonie.

Guiart Jean, 1963. La Chefferie en Mélanésie du Sud, Paris, Musée de l'Homme, Institut d'ethnologie.

LAGarde Louis, André-John OuETcho et Yves-Béalo GonY, 2008. Prospection archéologique sur le massif minier de Ouinné, Nouméa, rapport du département Archéologie du service des Musées et du Patrimoine de Nouvelle-Calédonie.

LeENHARDT Maurice, 1937. Gens de la Grande Terre, Paris, Gallimard.

Le Meur Pierre-Yves, 2010. La terre en NouvelleCalédonie: pollution, appartenance et propriété intellectuelle, Multitudes 41, pp. 91-98.

OuETCHO André-John, 2008. Inventaire préliminaire des sites archéologiques et traditions de la tribu de KWA (Commune de Thio), Nouméa, rapport du département Archéologie du service des Musées et du Patrimoine de Nouvelle-Calédonie. 
PARIS Jean-Pierre, 1981. Géologie de la NouvelleCalédonie : un essai de synthèse, Paris, Mémoire BRGM 113.

SAND Christophe, Frédéric Barp, David Baret et Béalo Gony, 2007. Relevé d'un abri sous roche sur l'emprise du chantier de Goro Nickel, Nouméa, rapport du département Archéologie du service des Musées et du Patrimoine de Nouvelle-Calédonie.

SAND Christophe, Jacques BoLÉ et André-John Ouetcho, 2003. Prehistoric Cultural Evolutions in a Melanesian Archipelago: the New Caledonia example, Antiquity 77 (297), pp. 505-519.

_, 2007. What were the real numbers? The Question of Pre-Contact Population Densities in New Caledonia, in P.V. Kirch and J.-L. Rallu (eds), The Growth, Regulation, and Collapse of Island Societies: Archaeological and Demographic Perspectives from the Pacific (chap. 15), Honolulu, University of Hawaii Press, pp. 306-325.
-, 2011. A Revision of New Caledonia's Ceramic Sequence, Journal of Pacific Archaeology 2, pp. 56-68.

SAND Christophe, Jacques BoLÉ, André-John OUETCHO et David BARET, 2008a. Inventaire archéologique sur le plateau du Camp des Sapins (mine SLN) - Commune de Thio, Nouméa, rapport du département Archéologie du service des Musées et du Patrimoine de Nouvelle-Calédonie.

—, 2008b. Parcours archéologique. Deux décennies de recherches du département Archéologie de NouvelleCalédonie (1991-2007), Nouméa, Les Cahiers de l'Archéologie en Nouvelle-Calédonie 17.

SAND Christophe et André-John Ouetcho, 1992. Bwede ko-tchon tchuvan-vare kein (des rivières déviées par les ancêtres). Premier inventaire archéologique de la commune de Yaté, province Sud de la Nouvelle-Calédonie, Nouméa, Les Cahiers de l'Archéologie en Nouvelle-Calédonie 1. 